\title{
La strada del salto nel vuoto
}

\author{
Elio GioAnola \\ Università degli Studi di Genova \\ egioanola@alice.it
}

\section{Resumen}

Il saggio si interroga sul realismo o neorealismo pavesiano e tenta di portare alla luce l'importanza definitiva dell'immobilismo ammirativa del mythos, forza pregnante di valenze ermeneutiche sempre presenti all'interno dell'opera di Pavese. Si scoprono così i «segnali dell'infinito», i «salti nel vuoto» dei testi pavesiani, specialmente di Feria d'agosto. Sono questi i segni ineludibili della grande modernità del autore. Il lavoro insiste ancora una volta sul profondo debito di Pavese riguardo il romanticismo e riguardo tutte le poetiche irrazionali del decadentismo e del simbolismo, i più ricchi modelli della scrittura, indubbiamente, novecentesca di Cesare Pavese.

Parole chiave: Realismo, neorealismo, logos, mythos, segnali dell'infinito, Leopardi, Pavese, Feria d'agosto.

The road of jump into the emptiness

\begin{abstract}
This essay questions the pavesian realism or neorealism and tries to show the definitive and admirative importance of the myth, a force full of hermeneutic valencies in Pavese's work. We discover in this way the "segnali dell'infinito», the "salti nel vuoto» in the pavesian texts, specially in Feria d'agosto. These are the unavoidable traces of the extreme modernity of this author. The article insists once more on the large debt of Pavese to the Romanticism and all irrational poetics od the Symbolism and Decadentism, without doubt, the richest models of the twentieth century writing of Cesare Pavese.
\end{abstract}

Key words: Realism, Neorealism, logos, mythos, segnali dell'infinito, Leopardi, Pavese, Feria d'agosto.

Gioanola, Elio. 2011. La strada del salto nel vuoto. Cuadernos de Filología Italiana, $\mathrm{n}^{\circ}$ extraordinario: págs. 197-207.

Ho avvertito in un misterioso e folgorante passaggio della prosa pavesiana, «la strada del salto nel vuoto», il possibile varco verso il cuore dell'opera dello scritto- 
re. La ricorrenza del termine vuoto è a dir poco ossessiva in questa scrittura: «Dietro il campo, una terra in salita, c'era il cielo vuoto» (Pavese 2002: 10); «Di là dagli steli alti ficcavo lo sguardo al vuoto del cielo», «A me basta quell'occhiata furtiva che ho detto, e il cielo vuoto si popola di colline e di parvenze» (Pavese 2002: 12); «Sull'orlo della balza che dava nel vuoto...»; «non si vedevano che le rampicanti azzurrine nel vuoto» (Pavese 2002: 23); «Finalmente sbucai. Vidi un'altra collina, e il cielo vuoto»; «La campagna diceva il suo vuoto» (Pavese 2002: 151). Bastano questi pochi rinvii a mettere in sospetto circa il realismo, se non addirittura il neorealismo, di Pavese, e non c'è bisogno nella circostanza di questo Convegno, che vede convocati i protagonisti della svolta critica sancita dall'apparizione del celebre numero 3/4 della torinese rivista Sigma (1964), di ribadire l'improprietà di quella definizione. La campagna di Pavese non dice presenze ma, appunto, il «suo vuoto», ed è in questa direzione che reputo sia utile scavare ancora, volendo puntare al fuoco centrale di un'ispirazione poetica drammaticamente totalizzante. Non che sia del tutto improprio ricorrere alla categoria del realismo, considerata la massiccia presenza dei riferimenti oggettuali, a partire proprio da quelli della campagna. Se infatti dico «la strada del salto nel vuoto» io so di avere a che fare con un preciso dato di realtà, perché il Salto sta sulla strada che da Santo Stefano porta a Canelli, di fronte alla falegnameria di Pinolo Scaglione, il Nuto della Luna e i falò, costruttore di bigonce per tutta la valle del Belbo. Si dice «salto» perché la strada in questo punto si affaccia su una riva in ripido e breve pendio, che dà sul piano dove scorre il torrente e passa la ferrovia per Alessandria. Dunque un massimo di precisione realistica, ma per un massimo di dissolvimento dei realia in un altrove assolutamente indeterminato, vuoto appunto. In un rapido sintagma lo scrittore sintetizza il nucleo centrale della sua poetica, che prevede bensì un reale, ma «incantato», come lui stesso dice. Di qui il lirismo fondamentale di una prosa sempre tentata di incatenare la sequenzialità narrativa del logos nell'immobilità ammirativa del mythos (il «mirare» che sostituisce, leopardianamente, il semplice «vedere»). Il salto toglie vista, anziché favorirla, come dovrebbe essere per chi guarda dall' alto verso il basso. Qui evidentemente lo sguardo, meglio l'«occhiata furtiva», è dal basso verso l'alto e allora la strada del salto funge da stacco dalla presenza topografica per l'accesso ad una dimensione altra. «Ficcavo lo sguardo al vuoto del cielo»: uno sguardo rivolto, anzi «ficcato», al vuoto non vede propriamente nulla, o tenta invano di vedere il non visibile. Quasi sempre il termine «vuoto», quando sia usato nella forma aggettivale, si accompagna al sostantivo «cielo», e il cielo è trasparenza, assenza di orizzonti e riferimenti oggettuali (le nuvole, tanto spesso evocate, non hanno nessuna consistenza di oggetto e accentuano, nonché ostacolare, la purezza del mezzo). Allora «vuoto» e «cielo» sono sostanzialmente la stessa cosa e rafforzano, congiungendosi, i significati loro deferiti. «C'era un cortile silenzioso e su per la scala che pareva scavata nella pietra mi fermai a guardare il cielo dalle finestrette» (Pavese 2002: 112); «Questo senso di fiducia [...] mi prende ogni volta che da un luogo chiuso do un'occhiata al cielo»; «è come in una stanza in cui da tempo non entra nessuno e la finestra è aperta al cielo» (Pavese 2002: 92); «Può darsi che la scala-finestra fosse quella della scuola [...], ma ciò che in essa contava e conta ancora è il cielo vuoto e immemoriale» (Pavese 2002: 135). Evidentemente la finestra ha la stessa funzione 
della strada del salto e permette il salto verso il vuoto altrove, che sta oltre il misurabile esperito dai sensi. Perché il vuoto si apra è necessaria la frapposizione di un oggetto-ostacolo che sia sull'orlo tra le due dimensioni, uno stelo di granturco, una vite, una fila di alberi, un profilo di collina. Ci vuole una presenza sull'estrema soglia per propiziare un passaggio 'di là': «Il roveto usciva da uno scoscendimento roccioso [un salto, appunto], e di là si vedeva il cielo». Questo jenseits der Dinge, di là dall'oggetto, punteggia costantemente la prosa pavesiana, specie nelle pagine per molti versi cruciali di Feria d'agosto: «scostavo le foglie taglienti, mi chinavo ai convolvoli, e di là dagli steli...» (Pavese 2002: 11); «Di là dalle piante sentivo gli uccelli che volavano basso» (Pavese 2002: 38); «di là dalle colline, è questione di tempo, c'è il mare» (Pavese 2002: 62); «di là dalle siepi si sentiva la vigna»; «ci piace guardare di là dalla siepe» (Pavese 2002: 101-102).

Sono sufficienti, credo, questi indizi per fare il nome nascosto, ma non tanto, sotto il loro pullulare, e il nome è quello di Giacomo Leopardi. Del resto è lo scrittore stesso a nominare esplicitamente il poeta a proposito di una delle figure messe in evidenza, quella della finestra:

So di un uomo che una semplice finestra di scala, spalancata sul cielo vuoto, mette in stato di grazia. Forse ci furono nella sua vita più finestre di scala che in un'altra? Perché di tutte le possibili figure d'infinito, scelse proprio questa? Ognuno è sensibile all'idea d'infinito, e già il Leopardi ne ha chiarito l'operazione, ma perché una finestra invece che una fila di piante o il profilo di una balaustra sul mare? (Pavese 2002: 134).

Chi non ricorda la «finestra passatoia» del grande recanatese? Ma citazioni leopardiane sul tema si possono cogliere a piene mani nello Zibaldone. Ne basti un paio:

Circa le situazioni che piacciono pel solo indefinito puoi vedere il mio idillio sull'Infinito, e richiamar l'idea di una campagna arditamente declive (la nostra strada del salto nel vuoto) in guisa che vista in certa lontananza non arrivi alla valle; e quella di un filare d'alberi, la cui fine si perda di vista, o per la lunghezza del filare, o perch'esso sia posto in declivio [...] Una fabbrica una torre [...] veduta in modo che ella paia innalzarsi sola sopra l'orizzonte, e questo non si veda, produce un contrasto efficacissimo e sublimassimo tra il finito e l'infinito $[\ldots] ;[\ldots]$ Piacciono quei luoghi dove la luce si confonde $[\ldots]$ con le ombre, come sotto un portico, in una loggia elevata e pensile, fra le rupi e burroni, in una valle, sui colli veduti dalla parte dell'ombra, in modo che siano indorate le cime [.... (Leopardi 1991).

Ma tra tutti i «segnali d'infinito», per usare le parole di Blasucci (1985) riferite a Leopardi, il più stringente è quello propiziato dalla siepe. Davanti al ragazzo scappato di casa per vedere il mare, che arriva sotto Cassinasco dopo avere salito la collina, ecco cosa appare a favorire il contrasto tra finito e infinito: «c'era una siepe di rovi che chiudeva la vista» (Pavese 2002: 70). E quell'altro ragazzo che va con la 
selvatica Sandiana, nel racconto che chiude Feria d'agosto, al santuario della Madonna della Rovere, così evoca il senso profondo di quella visita: «Una siepe di prugnole mi chiudeva l'orizzonte, e l'orizzonte sono nuvole, cose lontane...» (Pavese 2002: 164). Quasi una duplice citazione dei versi famosi: «e questa siepe, che da tanta parte / dell'ultimo orizzonte il guardo esclude». L'esclusione della vista è necessaria perché si schiuda il mondo della visione, quella che la mente, mirando, finge. Interdire la vista significa abolire la possibilità della misurazione, quella che permette l'orientamento tra gli oggetti del mondo. La vista è calcolo, anche intellettivo, come sa il greco antico in cui il perfetto del verbo vedere, orao, fa oida, io so; dunque escludere il guardo è anche rinunciare al pensiero cosciente, alla ragione deducente e sillogizzante. Di là dalla siepe è il vuoto dell'infinito, o del nulla, e ad esso ci si approssima chiudendo gli occhi, anche quelli della mente. Solo così possono dischiudersi, per un attimo estastico, gli interminati spazi, i silenzi sovrumani, la profondissima quiete.

Di cosa si parla, allora? Pavese che si rifà ad un modello consegnato agli archivi della storia letteraria? Un prosatore del Novecento che imita, quasi scopertamente, il più grande lirico del secolo precedente? La cosa non avrebbe molto senso, e nessuna relazione con la contemporaneità, se si trattasse di un problema di imitazione, per di più clamorosamente fuori tempo. Riprendo qui una frase di Pietro Citati che faccio mia senza esitazioni: «L'infinito è una meravigliosa malattia che fonda la modernità» (Citati 2008). Ho sempre pensato a Leopardi come a uno dei fondatori della modernità poetica, insieme al suo fratello disperatamente cittadino Baudelaire, non meno attratto del recanatese dall'abisso del nulla e dell'infinito: «Il n'est pas de pointe plus aerée que celle de l'Infini. Grand délice que celui de noyer son regard dans l'immensité du ciel et de la mer! Solitude, silence, incomparabile chasteté de l'azur». Questo cieco sguardo nell'immensità dell'infinito rappresenta la «vie suprème», in cui «le temps est disparu et c'est l'eternité qui règne». Non si dà modernità artistica se non in contrapposizione al progetto vincente dell'hegeliana Fenomenologia dello Spirito (Hegel 1967), il romanzo definitivo che tutto riconduce a conoscenza, unica vera storia che annichila tutte le storie inventate dai romanzieri e dai poeti. Ciò che evade dal cerchio dell'identità reale-razionale, dice Hegel nella Scienza della logica, non è, come vogliono i grandi romantici a cominciare da Hölderlin, «ciò che vi è di più elevato, di più vero, ma ciò che vi è di più insignificante, di meno vero» (Hegel 1981:156).

Quanto l'idealismo hegeliano abbia avuto il sopravvento sulle istanze irrazionalistiche avanzate dall'arte romantica, oltre che dal pensiero schopenhaueriano, è confermato dal trionfo degli storicismi e dei razionalismi di varia natura che hanno segnato la moderna storia della cultura occidentale. Pavese è cresciuto nella Torino illuministica, positivistica e storicistica di Gramsci, di Gobetti, di Augusto Monti, perfetta incarnazione pedagogica di homo gobettianus vulgaris, subito sentendosi un esemplare isolato di fauna 'decadente' in mezzo a tanta luce di ragione e ragionevolezza e kantiana eticità e sanità dello spirito (appena ventenne scriveva al suo maestro avverso: «Sana è in sé l'opera d'arte veramente buona ma non affatto per questo han da essere ugualmente sani il contenuto dell'opera e l'anima del creatore») (Gioanola 2003: 89). Insomma, fin dagli esordi Pavese è contagiato dalla 
«meravigliosa malattia» che lo trascina fuori dall'orto concluso e confortevole della razionalità e della storia, anche a costo dei risvolti di sofferenza psicopatologica cui va incontro.

Negli anni dei racconti e delle prose di Feria d'agosto, la scelta è precisa: «Il tuo problema è valorizzare l'irrazionale. Il tuo problema poetico è valorizzarlo senza smitizzarlo»; «Perché l'irrazionale solleva al tutti, all'universale?» (Pavese 2000: 245). Idea romantica. Ma è forse da dare ai cani per questo? Senza dubbio l'irrazionale è l'enorme reservoir dello spirito, come i miti lo sono delle nazioni. Le tue creazioni le trai dall'informe, dall'irrazionale, e il problema è come portarle alla consapevolezza»; «Lo stupore è la molla di ogni scoperta. Infatti esso è commozione davanti all'irrazionale - La tua modernità sta tutta nel senso dell'irrazionale» (Pavese 2000 : 246). Lo scrittore ha ben chiara la direzione verso cui cammina la modernità poetica, a cominciare dall'insurrezione del primo romanticismo e poi con le successive ondate simboliste, decadenti, espressionistiche, e dunque i richiami leopardiani sono l'esatto contrario dell'imitazione di un modello, ma un fecondo reimmergersi nelle fonti originarie.

Pavese è decisissimo a puntare «au fond de l'inconnu» perché sa, come Baudelaire che solo di là possono venire le scoperte più autentiche, non contaminate dalle sovrapposizioni del mondano normalizzato. Il giovane della prosa Una certezza deve stare rinchiuso più giorni in una stanza, prima insofferente poi via via più quieto, fino a quando una sera può uscirne. In questo momento, dice, «esitai un attimo sulla soglia. [...] Ebbene, ricordo che in quel momento di esitazione mi sentii appunto nel modo che ho detto - un grande stupore, un rincrescimento come di chi è trattenuto sull'orlo di un gesto, di un risveglio che stava avvenendo e adesso non avverrà più. Ma non fu come quando s'interrompe un'abitudine [...] bensì l'impressione di essere di colpo sbucato in un'aria tutta diversa dalla solita, un'aria che ti pare di avere dentro invece che intorno, un grande abisso d'aria, di vuoto, di possibili eventi e pensieri che sgorgherebbero dal più profondo te stesso, se questo te stesso non fosse subito sparito tant'era incredibile. Sono momenti questi, che si possono chiamare di disponibilità assoluta. S'intravede, dopo che uno li ha vissuti, che tutto il proprio passato visibile e perciò anche il presente e insomma tutta la vita, non conta per quello che si è fatto voluto sofferto ottenuto, e che tanto varrebbe starsene fermi in un angolo e [...] fissare a occhi chiusi questo stupore, quest'abisso» (Pavese 2002: 81-82). Viene da dire: «sedendo e mirando», con la conseguente abolizione del reale circostante e il salto, per un attimo, nell'abisso del vuoto che si spalanca oltre la soglia. E con l'imporsi del problema poetico per eccellenza: cosa dire se da una parte abolisco il mondo e la storia e la vita e dall'altra fisso l'inafferrabile nulla? Ma non è compito proprio ed essenziale della poesia quella che Lévinas chiama «l'indiscrezione riguardo all'indicibile»? E non è forse il problema posto e miracolosamente risolto da Leopardi nei quindici versi dell'Infinito?

Pavese trova una splendida figura per alludere a questa situazione non risolvibile in termini di logica: «Cose non dette trasparivano in fondo all'istante come un oggetto noto in fondo all'acqua di una vasca, e sarebbe bastato quel lieve coraggio di tuffare la mano, per toccare la lontana inafferrabile parvenza» (Pavese 2002: 86). Ma si tratta appunto di afferrare l'inafferrabile perché, appena la mano tocca la 
superficie dell'acqua, ciò che essa faceva intravedere, e nello stesso tempo nascondeva, dilegua. Sono parole della prosa Il tempo, in cui leggiamo anche: «Quanto a me, anche i ricordi più lontani mi coglievano come scoperte. Erano altrettanti risvegli che mi rimettevano nel presente. Il fatto più singolare - tanto che spesso lo provocavo ad arte - era di accorgermi che un gesto, un colore, una voce, li avevo già visti o sentiti chi sa quando, e che perciò risorgevano dalla mia stessa coscienza più che dalle cose intorno» (Pavese 2002: 86).

È stato detto giustamente che Pavese non è un caso di proustismo: in effetti il nostro scrittore non insegue il tempo perduto, ma semmai il non-tempo, e ciò significa che non fa collezione di ricordi collocabili in una durata cronologica o in un ambiente frequentabile dalla memoria volontaria o involontaria, ma evoca figure estreme, assolute, sciolte da ogni vincolo di tempo e spazio in quanto colte sull'orlo dell'abisso di nulla da cui sorgono e in cui si dissolvono. Semmai, ancora una volta, un caso di leopardismo: il grande poeta non evoca ricordi, ma ricordanze, o rimembranze, che non sono la stessa cosa, perché non si tratta di viaggi nella memoria, ma di ricerca della Cosa (das Ding, secondo Lacan) al di là delle cose, della quale le figure rievocate sono cenni, rinvii, luminose ipostasi.

Ecco allora l'unicità di queste figure, che tendono a perdere l'ambientazione, fondamento di ogni realismo, e a farsi assolute, come scrive lo scrittore nella celebre lettera del 1942 a Fernanda Pivano:

Deve pensare che immagini primordiali come a dire l'albero, la casa, la vite, il sentiero, la sera, il pane, la frutta ecc. Mi si sono dischiuse in questi luoghi [...] e rivedere perciò questi alberi, viti, sentieri $[\ldots]$ mi dà un senso di straordinaria potenza fantastica, come se mi nascesse ora, dentro, l'immagine assoluta di queste cose (Pavese 1966: 639).

È la stessa cosa che nella prosa Del mito, del simbolo e d'altro viene espressa in questo modo, con l'inevitabile riferimento all'intemporalità dell'universo infantile:

Così a ciascuno i luoghi dell'infanzia ritornano alla memoria; in essi accaddero cose che li han fatti unici e li trascelgono sul resto del mondo con questo suggello mitico. Ma il parallelo dell'infanzia chiarisce subito come il luogo mitico non sia tanto singolo, quanto quello di nome comune, universale, il prato, la selva, la grotta, la spiaggia, la casa, che nella sua indeterminatezza evoca tutti i prati, le selve ecc., e tutti li anima del suo brivido simbolico. Neanche nella memoria dell'infanzia il prato, la selva, la spiaggia sono oggetti fra i tanti, ma bensì $i l$ prato, la spiaggia come ci si rivelarono in assoluto e diedero forma alla nostra immagine (Pavese 2002: 126).

Ma un altro passo della medesima lettera alla Pivano ci riconduce al punto da cui siamo partiti:

Da questo salto non ero mai passato; si diceva allora che la strada proseguiva sempre a mezza costa, sempre affiancata da colline di così enorme estensione da apparire, viste sopra la spalla, come un breve orizzonte a fior di terra. Ero sempre arri- 
vato soltanto a quest'orizzonte, a questi canneti (capisce?). È come quando stesi nel prato, si guarda l'erba che chiude il cielo e sembra una foresta, ma presentivo di là del salto, a grande distanza dopo la valle che si espande come un mare, una barriera remota (piccina tanto è remota) di colline assolate e fiorite, esotiche. Quello era il mio Paradiso, i miei Mari del Sud, la Prateria, i coralli, Ophir, l'Elefante bianco ecc (Pavese 1966: 639).

Questa strada del salto evocatrice di un di là lontanissimo, vero paradiso perduto, è quella che percorrono i due ragazzi scappati di casa del racconto Il mare, alla ricerca di questa entità per eccellenza rappresentativa dell'altrove. Ricordiamo la definizione famosa di Pavese: «Vivere in un ambiente è bello quando l'anima è altrove. In città quando si sogna la campagna, in campagna quando si sogna la città. Dappertutto quando si sogna il mare». Essere altrove è decisivo soprattutto per quanto riguarda il mare: per trasfigurarsi miticamente il mare dev'essere un'assenza, quanto è lì davanti agli occhi, come nelle poesie scritte a Brancaleone, o nei romanzi brevi Il carcere e La spiaggia, diventa una presenza persino fastidiosa. Il racconto chiamato in causa è tutto ambientato in mezzo alle colline, tra le quali non c'è alcuna possibilità che il mare diventi una realtà esperibile.

Siamo nella raccolta Feria d'agosto, il racconto si svolge nel mese di agosto e il ragazzo di campagna, amico d'avventura del ragazzo di città in vacanza, si chiama Gosto. Siamo nel cuore della feria, cioè della sospensione dei lavori agricoli tra $i$ raccolti estivi e quelli autunnali, ma questa sospensione rinvia ad un'altra più assoluta, all'epoché che ferma il tempo e conduce alle esperienze estatiche, all' "unica grande estate» fissatasi immemorialmente negli stampi immaginativi dell'infanzia. Gosto dice che suo nonno ha visto il mare salendo in cima alle colline, il compagno sa che non può essere vero e così fantastica: «Io il mare l'ho sempre immaginato come un cielo sereno visto dietro dell'acqua». Il mare come un cielo, dunque una figura di altrove, d'infinito, del tutto indeterminata come quelle del vuoto o del nulla, di quanto insomma è di là. Chi del resto pensa a questo modo il mare è uno che della campagna non è parte, la custodisce dentro di sé come vivaio di immagini antiche ma non la vive attivamente, come il compagno: «Gosto non sa cosa sia mettersi davanti a una casa, e guardarla fin che non sembra più una casa» (Pavese 2002: 59). Infatti diventa la casa, un assoluto immaginativo come tante altre figure della campagna. $\mathrm{Ci}$ sono due modi per arrivare al mare da questa campagna, uno è quello che porta a valle, verso la pianura e poi verso la costa, ed è il modo delle cose reali, l'altro è quello che porta verso l'alto della collina, ed è il modo delle fantasie che aspirano a varcare i limiti della realtà. Ebbene, il primo modo è quello che si avvale, paradossalmente, proprio della strada del salto, che in questo caso non è nel vuoto: «Lo stradone che scende verso quelle colline [di Canelli] non è una strada di campagna; porta fuori della valle, in una pianura che scende sempre, che ha degli alberi che sembrano giardini. Già alla svolta, dopo lo sbocco nel vallone, dopo il ponte di ferro, c'è la casetta della Piana che ha un balcone di gerani. Laggiù non ci sono più vigne né boschi né stalle; di carretti tirati dai buoi non ne salgono di là; salgono invece i biroccini a tutta corsa e comitive coi parasoli» (Pavese 2002: 58). 
È la strada che porta in città, di cui Canelli è l'avamposto, appena oltre il confine che la separa dall'immobile universo della campagna. Dunque è la strada che va verso i luoghi della vita civile, della vegetazione ornamentale (gli alberi come giardini, i gerani), dell'attività e del buon vivere borghese (i biroccini, i parasoli), dei traffici, del tempo misurato dagli orari, della storia, del divenire, e dunque dei porti dove il mare è una presenza come un'altra. Quando i due ragazzi decidono la fuga nella sera in cui il fuoco ha distrutto, come un grande falò, una cascina nei presi di casa loro, pensano entrambi di andare verso il mare, ma resta ambigua la direzione, perché uno ha in testa il mare reale, l'altro quello immaginato come un cielo capovolto.

All'inizio imboccano la strada del salto ma già il buio della notte confonde le opposte mire, togliendo i riferimenti visivi e quindi creando una spazialità alternativa. Basta arrivare alla prima cascina sullo stradone che scende per immaginare un'altra direzione: «Dalla Piana passa la strada delle colline: di là dalle colline, è questione di tempo, c'è il mare. Bastava guardare in mezzo ai tronchi dei noci, tutta la valle scende laggiù. Passata la bassa del Belbo si è in altri paesi» (Pavese 2002: 62-63). Ed ecco l'effetto del buio sugli scappati di casa, o meglio su quello dei due, capace dei traslochi verso altrove: «Lo stradone con una svolta seguiva la costa, e dopo un poco non ci furono più dalla parte del salto gli alberi che facevano paura. Il ciglione della strada dava nel vuoto, sulla bassa piana del Belbo, che al lume delle stelle non si vedeva che nera. E anche le coste lavorate, che di giorno sono gialle, sembravano pozzi. Ci fermammo a guardare nel vuoto. Laggiù pareva che il vento attizzasse le stelle» (Pavese 2002: 64). Anche se la direzione è verso i "luoghi culti" di Canelli, la notte offre con lo schermo del buio la possibilità del salto nel vuoto. In ogni caso per il protagonista narratore quel viaggio notturno, che sembra avere come traguardo il mare a cui tutte le strade arrivano e da cui partono le navi, resta un'avventura che punta al non-dove e al lontano: «Riprendemmo la strada: Ma Gosto si mise a correre [...]. Allora gli corsi dietro per la strada che saliva, e più correvo più lui gridava, fin che fummo a un'altra svolta e qui si vide di nuovo, come un salto nel vuoto, la pianura e a gran distanza un cielo nero di colline» (Pavese 2002: 64). Ma la strada ormai non è più quella del salto: «Da un pezzo eravamo discesi in pianura, e andavamo fra i giardini e le ville» (Pavese 2002: 65). Ormai la notte è avanzata, i due fuggiaschi sono stanchissimi, vanno sotto un ponte del Belbo per stendersi sulle ghiaie, però il ragazzo che racconta continua a immaginare che «ad ogni svolta saremmo stati in riva al mare, quando poi ci fermammo e scendemmo sotto un ponte per dormire al coperto, mi pareva che il mare dovesse esistere soltanto di notte» (Pavese 2002: 65).

Al risveglio i ragazzi trovano vicino a loro il mendicante Rocco e con lui arrivano alla vicina Canelli e al tugurio dell'eremita. Gosto vorrebbe rimanerci, ma l'altro dice che non valeva scappare di casa per raccogliere l'erba ai conigli di Rocco. Avviene la separazione, il contadinotto ritorna a casa mentre il compagno decide di salire verso Cassinasco, inseguendo il suo miraggio. "Che cosa vuoi salire lassù», grida Gosto riprendendo lo stradone all'incontrario, «tanto il mare di là non lo vedi. Lo sapevo che il mare di là non si vede, l'avevo saputo fin da quando credevamo alle Ca' Rosse, ma con Gosto non l'avevo mai detto» (Pavese 2002: 68-69). Presa 
dunque la strada in salita, il protagonista cammina deciso sulla cresta della collina: «Adesso non m'importava più se di là da Cassinasco non avrei visto il mare. Mi bastava sapere che il mare c'era, dietro discese e paesi, e pensarci camminando tra le siepi. [...] Fermandosi, di là dalle siepi si sentiva la vigna e si vedevano i canneti. [...] Feci l'ultimo pezzo non pensando più a niente; c'era un siepe di rovi che chiudeva la vista. [...] Finalmente sbucai. Vidi un'altra collina e il cielo vuoto» (Pavese 2002: 69-70). In paese, dove c'è la festa e bruciano i falò, il ragazzo incontra Candido, il suonatore di clarino amico suo e della famiglia, che lo fa mangiare e dormire, dopo avere avvisato e tranquillizzato per telefono i genitori disperati per la scomparsa del figlio. Ed ecco il finale, dopo il risveglio nell'osteria di Cassinasco: «Dalla porta si vedeva la collina e un po' di cielo, e niente era più bello che pensarci adesso ch'ero con Candido e avevo parlato coi miei e nessuno sapeva che laggiù c'era il mare. La collina sembrava una nuvola e restava soltanto quel tronco di vite» (Pavese 2002: 73-74).

Che il mare sia la più classica delle figure d'infinito Pavese lo sa fin dal tempo in cui leggeva, da ragazzo, il capolavoro lirico leopardiano, lavorava alla tesi su Whitman, traduceva Moby Dick, scriveva il saggio su Melville e i versi dei Mari del Sud. In questo racconto, per me uno dei più belli della nostra prosa novecentesca, porta al massimo della significanza simbolica questa figura, corredandola di tutti quegli altri segnali che abbiamo evidenziato. Il mare, immaginato con gli occhi chiusi oltre le colline, è lontananza, interminato spazio, profondissima quiete e silenzio del non-tempo. Che lo scrittore miri da sempre, e con intensità ben consapevole a partire dalla scoperta mitica dei primi anni quaranta, a far vibrare la sua scrittura delle risonanze provenienti dall'extraterritorialità delle zone non soggette alla giurisdizione del reale-razionale, è cosa di tutta evidenza. Ma evidenziare tale evidenza è doveroso se non si vuole rimanere nell'inessenziale o farsi irretire dalle apparenze. Pavese è convinto che solo il «salto nel vuoto» porta alle soglie della verità ultima dell'esistere individuale e universalmente umano: «Sarà un discendere nella tenebra feconda delle origini dove ci accoglie l'universale umano, e lo sforzo per rischiararne un'incarnazione non mancherà di una sua faticosa dolcezza. Si tratta di cogliere nella sua estasi, nel suo eterno, un altro spirito. Si tratta di respirarne un istante l'atmosfera rarefatta e vitale» (Pavese 2002: 136). Sappiamo come lo scrittore faccia coincidere tali origini con l'infanzia, quando essa vive la verità delle sue radici etimologiche - in-fanzia da assenza del fari, cioè della parola -: da adulti e da poeti si tratterà proprio di dire quell'indicibile:

Di qui nasce che il più sicuro vivaio di simboli sia quello dell'infanzia: sensazioni remote che si sono sono spogliate, macerandosi a lungo, di ogni materia, e hanno assunto nella memoria la trasparenza dello spirito. Di qui nasce che agli ingegni contemplativi non si raccomanderà mai abbastanza di tapparsi i sensi davanti alla realtà e acontentarsi di quella che, filtrata dagli anni, riaffiora dal fondo della chiusa coscienza. L'illusoria ricchezza del reale non può essere giustamente valutata se non da chi sa che solo è nostro ciò che abbiamo posseduto sempre (Pavese 2002: 143). 
È la questione della «prima volta», inafferrabile perché hors signifié, ma più certa del cosiddetto reale se capace di nutrire le parole della «seconda volta».

Da bambini il mondo s'impara a conoscerlo non - come parrebbe - con immediato e originario contatto alle cose, ma attraverso i segni di queste: parole, vignette, racconti. Se si risale un qualunque momento di commozione estatica davanti a qualcosa del mondo, si trova che ci commoviamo perché ci siamo già commossi; e ci siamo già commossi perché un giorno qualcosa ci apparve trasfigurato, staccato dal resto, per una parola, una favola, una fantasia che vi si riferiva e lo conteneva. [...] Rigorosamente, non esiste un «veder le cose la prima volta»: quella che conta è sempre una seconda (Pavese 2002: 143).

Ancora Leopardi, inevitabilmente:

Da fanciulli, se una veduta, una campagna, una pittura, un suono ecc. un racconto, una descrizione una favola, un'immagine poetica, un sogno, ci piace e ci diletta, quel piacere e quel diletto è sempre vago e indefinito: l'idea che ci desta è sempre indeterminata e senza limiti: ogni consolazione, ogni piacere, ogni aspettativa, ogni disegno illusione ec. (quasi anche ogni concezione) di quell'età tien sempre dell'infinito: e ci piace e ci riempie l'anima indicibilmente, anche mediante i minimi oggetti (Leopardi 1991).

Insomma, l'oggettualità di quanto pervenuto alla parola e alla figurazione, o alla seconda volta, si carica di senso se intriso delle ricordanze infantili, dell'immagine antica, del vago e indefinito della prima volta.

Ma non ci si abbandona così ad una pericolosa attrazione del nulla? Cosa sono vuoto, buio, abisso se non un puro niente? «Il ragazzo saliva per questi sentieri, vi saliva e non pensava a ricordare; non sapeva che l'attimo sarebbe durato come un germe [...]. Forse quest'attimo era fatto di nulla, ma stava proprio in questo il suo avvenire. Un semplice e profondo nulla, non ricordato perché non ne valeva la pena, disteso nei giorni e poi perduto, riaffiora davanti al sentiero, alla vigna, e si scopre infantile, di là dalle cose e del tempo» (Pavese 2002: 140-141). Pavese è stato a lungo guardato con qualche sospetto per le supposte ombre di nichilismo decadente aleggianti sulla sua opera. Ma il nulla pavesiano non è affatto nichilistico perché non ha fondamenta metafisiche, ma è custode e fonte di tutto il possibile, motore increato di ogni creazione. Una parola che non rechi traccia di questo nulla è puro strumento di comunicazione, nuda denotazione, senza echi di rimembranza e lontananza e anche speranza, soli capaci di fecondare una scrittura che voglia essere realmente poetica. Per arrivare al cuore dell'opera pavesiana bisogna percorrere senza esitazione la strada del salto nel vuoto. 


\section{Bibliografia}

BLASUCCI, Luigi (1985): Leopardi e i segnali dell'infinito. Bologna, Il Mulino.

CitATI, Pietro (2008): La malattia dell'infinito. Le letteratura del Novecento. Milano, Mondadori.

GioAnola, Elio (1971): Cesare Pavese. La poetica dell'essere. Milano, Marzonati Editore. GiOANOLA, Elio (2002): «Introduzione» a Feria d'agosto. Torino, Einaudi, pp. v-xxii.

GioAnola, Elio (2003a): Cesare Pavese: la realtà, il silenzio, l'altrove. Milano, Jaka Book.

Gionnola, Elio (2003b): «Pavese e il silenzio», Cuadernos de Filología Italiana, 9, pp. 121-138.

HEGEL, Georg Wilhelm Friedrich (1967): Fenomenologia dello spirito, a cura di Enrico De Negri. Firenze, La Nuova Italia.

HEGEL, Georg Wilhelm Friedrich (1981): Scienza della logica, a cura di Valera Verra. Torino, UTET.

LACAN, Jacques (2002): Scritti. Torino, Einaudi.

LEOPARDI, Giacomo (1991): Zibaldone di pensieri, a cura di Giuseppe Pacella. Milano, Garzanti.

LÉVINAS, Emanuele (2007): Il tempo e l'altro. Genova, Nuovo Melangolo.

PAVESE, Cesare (1966a): Lettere 1924-1940, vol. I, a cura di Lorenzo Mondo. Torino, Einaudi.

PAVESE, Cesare (1966b): Lettere 1945-1950, Vol. II, a cura di Italo Calvino. Torino, Einaudi.

PAVESE, Cesare (1998): Tutte le poesie, a cura di Mariarosa Masoero. Torino, Einaudi.

PAVESE, Cesare (2000): Il mestiere di vivere. Diario 1935-1950, a cura di Marziano Guglielminetti e Laura Nay. Torino, Einaudi.

PAVESE, Cesare (2002): Feria d'agosto, a cura di Elio Gioanola. Torino, Einaudi. 